\title{
A prática de ensino como formação docente do professor de matemática
}

The practice of teaching as teacher training of the math teacher

Flávio Nazareno Araújo Mesquita ${ }^{1}$

Renato Borges Guerra ${ }^{2}$

\section{Resumo}

Este texto tem por objetivo tratar do problema da formação pessoal do professor a partir da noção de relação com o saber sob a compreensão da teoria antropológica do didático. Um dispositivo teórico-metodológico é desenvolvido para proporcionar um quadro de análise que possibilite evidenciar mudanças da relação do professor com um saber matemático escolar. Resultados obtidos a partir da análise da construção de compreensões, sobre a resolução da equação do segundo grau, mostram a potencialidade do dispositivo de formação pessoal por revelar aquisições de novos saberes e procedimentos úteis, bem como novos problemas relacionados, para as práticas de ensino do professor.

Palavras chave: formação de professores; transposição didática interna; percurso de estudo e pesquisa; equação do segundo grau.

\section{Abstract}

This paper aims to address the issue of the personal formation of the teacher from the notion of relation with knowledge under the understanding of the anthropological theory of the didactic. A theoretical-methodological device is developed to provide a framework of analysis which may allow evidence of changes in the teacher's relationship with a school mathematical knowledge. Results obtained from the analysis of the construction of understandings on the second degree equation's resolution show the potential of the personal training device for revealing acquisitions of new knowledge and useful procedures as well as new problems related to the teaching practices of the teacher.

Keywords: teacher training; internal didactic transposition; route of study and research; second degree equation.

\footnotetext{
${ }^{1}$ Universidade Federal do Pará | flavio.nam@hotmail.com

${ }^{2}$ Universidade Federal do Pará | rguerra@ufpa.br
} 


\title{
Introdução
}

A Teoria Antropológica do Didático - TAD - (CHEVALLARD, 1999; CHEVALLARD, et al., 2001, CHEVALLARD, 2009a) segue em continuação da Teoria da Transposição Didática TTD - (CHEVALLARD, 1985, 1991) e ambas se inserem no programa epistemológico de pesquisa que situa a modelagem da atividade matemática no núcleo dos fenômenos didáticos (GASCÓN, 1999, 2003).

Assim, o ensino e a aprendizagem do conhecimento matemático são assumidos como uma atividade humana situada, que se realiza no interior de instituições concretas, e, portanto, dotada de uma "razão de ser" ou de um "tipo de racionalidade" que Ihe dá sentido. Isso inclui as condições, nem sempre visíveis, impostas por essas instituições podendo, inclusive, limitar o ensino e a aprendizagem desse conhecimento.

Como desdobramento dessa compreensão, a TAD insere as atividades matemáticas da escola em um domínio, mais amplo, de estudo das práticas matemáticas institucionalizadas.

\begin{abstract}
A abordagem epistemológica considera que qualquer problema didático contém algumas atividades matemáticas que estão sendo produzidas, ensinadas, aprendidas e praticadas. Alinhado a isto, essas atividades matemáticas acontecem em uma instituição concreta (geralmente, uma educacional), e a sua forma de existência e sua evolução dependerão principalmente das restrições educacionais relacionadas com o processo de transposição didática (BOSCH; CHEVALLARD; GASCÓN, 2006, p. 3-4, itálico do autor, tradução nossa).
\end{abstract}

A forma de vida e evolução de uma prática matemática depende do processo de sua transposição didática, ou seja, de sua reconstrução, em que se consideram seus usos, para torná-la possível de ser ensinada, a fim de que seja aprendida. E mais, esse processo está submetido a várias condições - que podem até mesmo limitar o ensino e a aprendizagem dessa prática - originadas na escola, na pedagogia, além das originadas na sociedade, na cultura e na humanidade.

Assim, quando um professor está frente a uma prática da matemática escolar, ele pode pensá-la como uma transposição de uma prática matemática de outra instituição, em particular, como prática da matemática acadêmica, como os formadores de professores de matemática costumam fazer crer.

Mas, sob a égide da TAD, ele poderá pensá-la também como uma transposição didática de práticas de outras instituições escolares ou das práticas das ciências aplicadas, como a economia e a engenharia, ou ainda, como transposição de práticas não eruditas, como a dos comerciantes e artesãos, por exemplo, em que se insere historicamente a regra de três (SILVA, 2017).

A noção de transposição didática se constitui, assim, o cerne da TAD, pois seu objeto principal de pesquisa é a ação do homem em situação com matemática, em particular, o modo como essa atividade é concebida e institucionalizada mediante suas práticas.

Para o estudo de seu objeto de interesse, a TAD propõe um modelo mínimo chamado de praxeologia, que é denotado $[T / \hat{O} / \Theta / \Theta]$, e, que em unidade, se constitui de dois blocos: o primeiro é o da práxis, constituído daquilo que se faz - denominado tarefa $T$ - e de como se faz essa tarefa - denominado técnica ô; o segundo, é composto de um discurso da instituição que descreve, explica ou justifica essa técnica - a tecnologia $\theta$ - e de uma teoria $\Theta$, ou discurso institucional, que fundamenta essa tecnologia. 
A noção de praxeologia se insere na noção de transposição didática, no sentido de que, o que é transposto de uma instituição à outra são as praxeologias, ou fragmentos delas. Então, uma organização didático-matemática, que vive em uma instituição de ensino, sob essa compreensão, é uma rede articulada e integrada de praxeologias segundo a orientação de um saber, não necessariamente ali explícito.

Essa compreensão é depreendida a partir do seguinte modelo epistemológico lato sensu anunciado por Chevallard (1999).

As organizações pontuais vão assim se agregando, primeiramente como
organizações locais, $\left[T_{i} / \hat{O}_{i} / \theta / \Theta\right]$, centradas sobre uma tecnologia $\theta$
determinada, em seguida em organizações regionais, $\left[T_{i j} / \hat{O}_{i j} / \theta_{j} / \Theta\right]$,
formada em torno de uma teoria $\Theta$. (Além disso, nomeamos uma
organização praxeológica global o complexo praxeológico $\left[T_{i j k} / \hat{O}_{i j k} / \theta_{i k} / \Theta_{k}\right]$
obtido em uma determinada instituição pela agregação de várias
organizações regionais correspondentes a várias teorias $\Theta_{k}$ ).
(CHEVALLARD, 1999, p. 5)

Ravel (2003) em sua pesquisa considera a resposta R ao problema praxeológico concreto do professor por meio da TDI em suas duas fases, mas dirigindo seus questionamentos ao papel do professor e dos autores de livros didáticos na evolução da transposição didática de um saber. Seu olhar se distancia da TDI como um processo problemático e pessoal de um professor concreto em encontrar uma resposta $\mathrm{R}$ como reconstrução de uma OM concreta em uma instituição concreta.

Este trabalho, tomando a TDI de modo distinto de Ravel (2003), ou seja, o de encontrar a resposta $\mathrm{R}$ de reconstrução de uma $\mathrm{OM}$ como um tipo de tarefa problemática a ser enfrentada por cada professor, questiona se a questão $Q_{0}$ pode ser tomada como um Problema de Formação Pessoal do Professor - PFPP - que, Chevallard (2009a) anuncia como o problema de encontrar as condições que permitam ao professor mudar sua relação com um dado saber escolar, aqui entendida como mudança de seu equipamento praxeológico e suas relações, que inclui as situações, relativas a esse saber.

Assim, realizar uma transposição didática consiste em recriar uma organização articulada e integrada de praxeologias, o que inclui fragmentos praxeológicos, para atender uma intenção de ensino, pois sob a TAD, o saber a ser ensinado necessita responder uma ou mais questões, matemáticas ou extramatemáticas, que lhe deem sentido, e isto implica em reconstruir organizações de tarefas da matemática escolar que atendam a essa intenção de ensino.

Então, uma questão se põe frente ao professor e é anunciada por Chevallard (2002, 1998a, 1998b) como problema praxeológico do professor, ou ainda, como o de ele encontrar uma resposta à questão seguinte $Q_{0}$ : o que ensinar "desse saber" e como ensinar?

A busca de respostas à questão Qo, relativas aos saberes da matemática escolar, encaminha uma das linhas de investigação à luz da TAD que considera os saberes da epistemologia e da história da matemática, inclusive dos saberes que funcionam com matemática, não necessariamente nessa ordem ou de modo simultâneo. Pois, essa infraestrutura de saberes, pode permitir construir uma epistemologia artificial para o saber considerado, que é chamada de modelo epistemológico de referência - MER - como uma fecunda trajetória de praxeologias. 
Essa linha de investigação encaminhou outros problemas, entre eles, o problema do currículo (CHEVALLARD, 2009a) e, relacionado a este, o problema da desarticulação das práticas matemáticas escolares ((BOLEA (2003); FONSECA (2004); SIERRA (2006); BARQUERO, BOSCH, GASCÓN (2007); GARCIA, et al., (2006); BARQUERO (2009); ANDRADE (2012); GUERRA, ANDRADE (2013); ASSUNÇÃO (2016), SILVA (2013), SILVA (2017); FONSECA, BOSCH, GASCÓN, (2010); LUCAS, et al. (2014)) por evidenciar, por exemplo, que as praxeologias pontuais são marcadas por tarefas e técnicas isoladas, que não se desdobram em outras tarefas.

Essa abordagem também levou ao encontro da inevitável relação entre a questão $Q_{0}$ e a formação de professores, considerando que, em geral, a reconstrução de práticas com matemática da escola, exigida pela questão $Q_{0}$, não se faz presente nos currículos de formação de professores, pois a Matemática, não raramente, é tomada pelos formadores como única e inquestionável.

As investigações realizadas por Chevallard (2009a), Gascón (2010, 2014), e Lucas, et al. (2014), tomam a questão Qo tendo como instituição uma comunidade docente e, como tal, encaminham respostas a partir de um percurso de estudo e investigação ou pesquisa PEP ${ }^{3}$ (CHEVALLARD, 2009a, 2009b) sob condições impostas por essa instituição e, talvez por isso, ignorem a dimensão pessoal do professor no enfrentamento desse tipo de problema.

No entanto, Chevallard (2009a) não descarta a dimensão pessoal do questionamento Qo quando considera as compreensões do que ele denomina de antropologia cognitiva (CHEVALLARD, 2005), exposta no trecho a seguir:

Sob o quadro da TAD, uma pessoa x é o resultante de seu passado e presente de conformidades institucionais. $O$ conhecimento de uma pessoa $x$ pode assumir duas formas principais. Em diacronia, podemos imaginar 0 fazer da história da pessoa como sujeito, por meio da crônica de suas conformidades e contra conformidades. Em sincronia, podemos imaginar o quadro de suas relações pessoais, que é dizer o que eu chamei acima de seu universo cognitivo UC (x) [...]. Mas também podemos encarar $\mathrm{O}$ conjunto de praxeologias que a pessoa dispõe, ou que está equipada (mesmo que ela não possa atualizar tal ou tal praxeologia que venha a ocupar tal posição dentro de tal instituição): é o que chamo de equipamento praxeológico da pessoa. Claro que, mesmo que haja uma história da pessoa como sujeito, existe uma dinâmica cognitiva, que é o que faz com que alguns objetos desapareçam da UC (x), enquanto outros irão aparecer, e há uma dinâmica praxeológica em que o equipamento praxeológico de $\mathrm{x}$, que denotamos por EP $(\mathrm{x})$, muda - algumas partes deste equipamento perdem suas características de operação, enquanto outras partes são remodeladas e novos elementos são adicionados ao longo do tempo (CHEVALLARD, 2009a, p. 6-7, tradução nossa).

A dimensão pessoal da questão do tipo $Q_{0}$ se impõe quando se observa que a construção de uma resposta demanda dinâmicas cognitivas e praxeológicas da pessoa do professor. Além disso, essa dimensão se impõe também considerando que a dimensão

\footnotetext{
${ }^{3}$ PER no original (Parcours d'Étude et de Recherche).
} 
institucional se desenvolve em dialética com a dimensão pessoal, ou seja, não há instituição sem pessoas. Essa compreensão é deixada clara no seguinte extrato de texto.

Existe uma dialética entre as relações institucionais e pessoais; as primeiras proporcionam as condições e restrições sob as quais se criam e evoluem as segundas; as segundas são pontos de apoio para as primeiras quando alcançam um estado idôneo segundo as exigências institucionais (ARAYA; MATHERON, 2007, p.262).

Não queremos, assim, afirmar a dispensabilidade do enfretamento da questão $Q_{0}$ pela instituição docente, mas destacar a indispensabilidade da dimensão pessoal dessa questão para a formação do professor. Chevallard deixa clara tal dimensão quando afirmar que: "Existe o didático quando um sujeito $Y$ tem a intenção de fazer nascer ou mudar, de certa maneira, a relação de um sujeito $X$ com um objeto $O$. (Naturalmente pode ocorrer que $Y=X$ )" (CHEVALLARD, 2005, p.150, grifo nosso).

Assim a questão do tipo Q é um problema didático concreto que um professor em particular enfrenta no exercício de sua profissão. Essa concretude se substancia na diversidade de condições impostas pelos diferentes níveis de codeterminação didática ${ }^{4}$ sobre as atividades docentes, entre elas, mas não somente, dos diferentes sistemas de ensino, pedagogias e escolas, por exemplo, a EJA (Educação de Jovens e Adultos), o ensino profissional e as oriundas da educação do campo. Estas, em particular, não deixam claro para o professor o que e como ensinar para atender às especificidades dessas escolas e pedagogias, como mostra o estudo da Pedagogia da Alternância por Assunção (2016).

É sob esse olhar também que se faz emergir esse tipo de questão Q como problema concreto para um dado professor. É na busca de responder essa problemática que ele se torna agente, geralmente esquecido, do processo de transposição didática.

Sobre a atividade desse sujeito-agente da transposição didática é que direcionamos nosso olhar. Mais especificamente, as atividades realizadas em modo contínuo nos processos mais internos das transposições didáticas dos saberes que serão ensinados pelo professor, que são chamadas de transposições didáticas internas, daqui em diante TDI. Parafraseando Charlot (2000) sobre a relação com o saber, diríamos dos momentos em que ele conversa com o mundo, com os outros, e de modo dominante consigo, em busca de colocar o saber em seu próprio e eminente discurso para ser ensinado em sala de aula.

Essa compreensão sobre a questão $Q_{0}$ é anunciada como um problema de cada professor no processo da TDI para o enfretamento de uma situação de ensino de um saber específico, para uma posição específica, ano ou série, de uma escola específica, que pode ser depreendida do seguinte extrato de texto.

Podemos falar, então, de uma praxeologia didática do professor relativa a uma organização matemática OM concreta. É a resposta $R_{\pi}=\left[T_{\pi} / \tau_{\pi} / \theta_{\pi} / \Theta_{\pi}\right]$ que cada professor dá ao problema de reconstruir uma OM concreta em uma instituição concreta (BOSCH; GASCÓN, 2001, p. 2-3, grifos do autor, tradução nossa).

\footnotetext{
${ }^{4}$ Ver La TAD face au professeur de mathématiques (CHEVALLARD. 2009)
} 
Ravel (2003) em sua pesquisa sobre práticas docentes considerou a TDI, mas com foco no produto desta, a resposta $\mathrm{R}$ praxeológica, sem considerar o processo descrito por Chevallard (2009a) com fundamento na antropologia cognitiva que busca encontrar as condições que levam a esta resposta.

Esta pesquisa considera a TDI de modo distinto de Ravel (2003), ou seja, que o processo de encontrar a resposta $\mathrm{R}$ de reconstrução de uma $\mathrm{OM}$ é um tipo de tarefa problemática a ser enfrentada por cada professor encaminha que o processo de construção de uma resposta para $Q_{0}$ pode se constituir em um Problema de Formação Pessoal do Professor - PFPP - no sentido dado por Chevallard (2009a) para o problema de formação do professor, como o de encontrar condições que permitam ao professor mudar sua relação com um dado saber escolar, aqui entendida como mudança de seu equipamento praxeológico e suas relações, que inclui as situações, relativas ao saber considerado.

Assim, nesta pesquisa, especificamente, questionamos: o estudo para a reconstrução de um modelo epistemológico de referência relativo a um dado saber, restrito às práticas possíveis de serem realizadas numa escola, constitui condição favorável para a mudança de relação de um professor quando este busca responder à questão do tipo $Q_{0}$ sobre esse saber? Mais geralmente, o estudo de um modelo epistemológico relativo a um dado saber torna a questão $Q_{0}$ um PFPP? Nosso objetivo é tratar do problema da formação pessoal do professor a partir da noção de relação com o saber sob a compreensão da teoria antropológica do did

Na sequência, consideramos os recursos teóricos e metodológicos, as análises e resultados encontrados frente ao problema de reconstruir modelos epistemológicos artificiais e OM associadas sobre a resolução da equação do segundo grau, sob as condições iniciais anunciadas, com objetivo de encontrar evidências de mudanças de relação de um professor com esse saber, ou seja, mudanças nas organizações matemáticasdidáticas, o que inclui seu modo de pensar, sobre esse objeto.

\section{Recursos teóricos e metodológicos}

O caminhar teórico da TAD demandou a noção de percursos de estudos e investigação ou pesquisa - PEP - com objetivo de fundamentar as organizações didáticas, tanto as escolares como de qualquer outro tipo de instituição, em uma epistemologia realmente funcional, na qual os saberes apareçam como "maquinas" produtoras de conhecimento úteis a criação de respostas R a questões Q. (BOSCH; GASCÓN, 2010, p.82).

Em particular, O PEP tem destacada relevância como percurso de formação de professores como destaca, por exemplo, Chevallard (2009a, 2009b, 2012) e Gascón (2014), entre outros, o que o credencia para atingir nosso objetivo. Desse modo, sob essa compreensão, buscamos evidenciar que o enfrentamento pessoal da questão $Q_{0}$ pode se constituir em um PEP para formação pessoal do professor.

Chevallard $(1991,2005)$ divide o processo da transposição didática em duas etapas: a transposição didática externa, daqui em diante TDE, realizada pela noosfera que compreende a transposição didática realizada com participação de diferentes agentes da sociedade - tais como as autoridades e especialistas da educação, inclusive de pais e professores, e a TDI realizada em duas fases pelo professor (Ravel, 2003): a elaboração do texto do saber e o ensino propriamente dito desse saber. 
O texto do saber, que se constitui em uma organização praxeológica para o ensino de um saber, é construído pelo professor em iterações com sua relação com o saber a ser ensinado, ou seja, a partir de sua história de vida com as praxeologias em situação de ensino ou estudo, em diferentes instituições de ensino em que atua e atuou como professor, como professor-aluno em formações continuadas e até quando aluno em formação nos diferentes níveis de ensino.

Portanto, o processo de TDI se desenvolve segundo uma compreensão do professor sobre o saber a ser ensinado que denominamos aqui de Modelo Epistemológico Pessoal de Referência - MEPR - que pode ser mudado ou não durante o desenvolvimento de seu texto de saber.

Os encontros do professor com essas praxeologias se deram, e se dão em condições distintas, nem sempre visíveis por ele, em processos de ensino ou estudos propriamente ditos, que são denominados por Chevallard $(1999,2005,2009 b)$ de sistemas didáticos e denotados por $S(P, A, O)$, que unem em relações distintas os encontros do professor $P$ e dos alunos $A$ com o saber $O$. Nesses tipos de sistemas didáticos também se encontram os sistemas didáticos solitários, em que $\mathrm{A}=\mathrm{P}$, os denominados sistemas autodidáticos em que a mesma pessoa ocupa os lugares de professor e aluno simultaneamente (CHEVALLARD, 1992, 1999, 2005, 2009b, 2011).

Seguindo a compreeensão da TAD, podemos pensar a diacronia da TDI em duas fases. A primeira fase se desenvolve por meio de um sistema didático solitário $S(P, Q)$ que mobiliza sincronicamente praxeologias, e fragmentos destas, sujeitas a uma diacronia escolar, ou seja, as praxeologias aprendidas que participam da história de vida do professor e as praxeologias delas decorrentes, com ajuda do estudo, como saberes aprendidos por meio da sincronia das diacronias de práticas e de obras até então estudadas pelo professor, ou seja, a partir se $S(P, Q)$ um novo sistema solitário $S(P, O)$ de estudo e análise é instaurado para a manutenção ou construção de um novo MEPR que permitirá confirmar ou mudar a resposta $R$, que não sendo definitiva, denotamos agora por $R^{\diamond}$.

A resposta $R$ é construída na primeira fase com base no modelo didático de referência lato sensu proposto por Chevallard (1999) e é constituído de seis momentos didáticos: 1) momento do encontro com a praxeologia; 2) momento exploratório; 3) momento de estudo e construção do entorno tecnológico-teórico; 4) momento do trabalho da técnica; 5) momento da institucionalização, 6) momento da avaliação - com ênfase em quatro desses momentos, os momentos didáticos dois, três, quatro e cinco, que são aqui traduzidos, respectivamente, como momentos de naturalização, problematização, reconstrução e institucionalização da técnica em jogo no MEPR.

Essa resposta $R$ é sempre tomada como provisória, então se inicia a segunda fase da TDI que consiste em por à prova empiricamente por meio da exposição de $R^{\diamond}$ em um novo sistema didático, chamado de sistema didático auxiliar. Esses sistemas didáticos auxiliares podem ser instaurados em uma sala de aula com alunos $S\left(P, A, R^{\diamond}\right)$ ou em encontros com colegas docentes, inclusive em formação inicial, $S\left(P, P^{\prime}, R^{0}\right)$, e, não menos importante, com um professor orientador, $S\left(P, P^{\prime \prime}, R^{0}\right)$.

Os questionamentos $\mathrm{Q}_{i}$ oriundos dos sistemas didáticos auxiliares, independente do agente questionador, aluno, colega docente, orientador, inclusive o próprio professor agente da TDI, leva à instauração de um novo sistema solitário que reinicia a primeira fase da TDI. 
A reiteração desse processo busca o encontro de uma resposta $R$ que esteja dentro do que o professor julga admissível como prática matemática da escola, segundo os programas oficiais, inclusive a respeito do tempo de ensino necessário, em consonância com a intenção didática do professor de ensinar um saber, em conexão com outros sabres, de modo a dar sentido a essa resposta $\mathrm{R}$ encontrada.

Essa reiteração das duas fases da TDI constitui a diacronia de um processo de estudo e investigação ou pesquisa (PEP) que pode ser representado esquematicamente como segue.

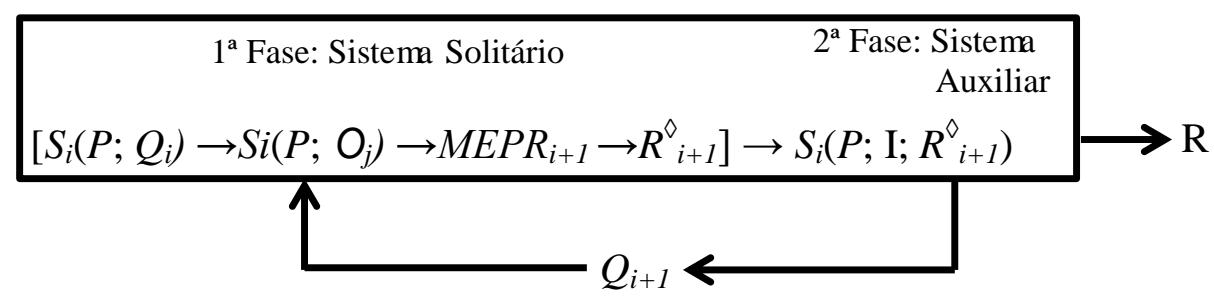

Em que I denota os sujeitos que interagem com o professor $\mathrm{P}$ no sistema auxiliar, como alunos $\mathrm{A}$, colegas $\mathrm{P}^{\prime}$, e orientador $\mathrm{P}^{\prime}$, por exemplo, e $\mathrm{O}$ a obra estudada, entendida como textos teóricos e respostas prontas $\mathrm{R}^{\diamond}$ já existentes.

$O$ processo iterativo dado pelo esquema acima nos diz que a resposta $\mathrm{R}^{\diamond}$ é a resposta construída, e não definitiva, pelo sistema didático solitário $S_{i}(P ; O)$ que encaminha o estudo de obras e respostas prontas instaurando outro sistema didático solitário $S_{i}\left(P_{i} Q_{i}\right)$ das análises teóricas das $R^{\diamond}{ }_{k}$ até então encontradas. Essa resposta $R_{i+7}^{\diamond}$ é então difundida e defendida pelo professor $P$ no sistema didático auxiliar $S_{i}\left(P ; l ; R_{i+7}^{\diamond}\right), e$, quando sucumbe a um questionamento $Q_{i+1}$ julgado pertinente por $P$, o desenvolvimento da TDI gera, então, um meio $M$ esquematicamente representado por $M=\left\{M E P R_{0}, M E P R_{1}, \ldots, M E P R_{k, 1} R_{1}^{\diamond}, R_{2}^{\diamond}, \ldots\right.$, $\left.\mathrm{R}^{\diamond}{ }_{k}, \mathrm{Q}_{0}, \ldots, \mathrm{Q}_{\mathrm{m}}, \mathrm{O}_{0, \ldots,} \mathrm{O}_{p}\right\}$ que passa a constituir também as condições de construção de uma resposta $\mathrm{R}$ pelo professor.

Esta metodologia encaminha uma investigação dita completa por Chevallard (20122013), pois implica a realização dos seguintes "gestos" básicos realizados não necessariamente de modo linear:

Observar as respostas $R^{\diamond}$ que vivem nas instituições.

Analisar - notadamente em duplo plano experimental e teórico- essas respostas $R^{\diamond}$.

Avaliar essas mesmas respostas $R^{\diamond}$.

Desenvolver uma resposta própria, $\mathrm{R}$.

Difundir e defender a resposta $\mathrm{R}$ assim produzida.

Segundo Chevallard (2012-2013) quando P simplesmente "copia" - isto é, retoma sem alterações - uma resposta existente, $R^{\diamond}$, como uma resposta $R$, então, nesse caso, há em estado "puro", a condição de P não ter que, de qualquer forma, apresentar para comentar, analisar, e especialmente para defender sua resposta, ou seja, não há investigação e pesquisa.

As características desta metodologia, aqui descritas, nos permitem afirmar que ela é o seguinte PEP:

$$
\left[S\left(P ; Q_{0}\right)\right] \gg\left\{M E P R_{0}, M E P R_{7}, \ldots, M E P R_{k}, R_{0}, R_{1}, \ldots, R_{n}, Q_{0}, \ldots, Q_{m}, O_{0}, \ldots, O_{p}\right\} \rightarrow R
$$

De outro modo o sistema didático $S\left(P ; Q_{0}\right)$ instaura o PFPP, cuja resposta é fundamentada pelo meio M construído. Assim, a resposta $R$ pode ser explicada, justificada e até produzida a partir dos $M E P R_{i}$ e das questões $Q_{i}$ que levaram às modificações transitórias de $R_{i}$ ou MEPR . 
O desenvolvimento do PEP é tomado de forma finalizada quando o profesor encontra um MEPR, admissível por ele, que atenda tanto sua intenção didática como a da escola, em conformidade com o programa oficial da disciplina, e, com tempo de ensino compatível com o preconizado por essa escola, para o objeto de ensino em questão.

As reconstruções de MEPR, que fundamentam a resposta $R$, indica a mudança de relação do professor com o saber em jogo, e, portanto, sob as condições aqui expostas, pressupomos que a TDI possa ser tomada como uma resposta à dimensão pessoal do problema praxeológico do professor, ou simplesmente, PFPP.

Além disso, o meio $\mathrm{M}$ pode permitir a construção de diferentes respostas para $\mathrm{O}$ problema praxeológico pessoal do professor com relação a um dado saber. De outro modo, a construção de uma resposta $\mathrm{R}$ pode ser expressa como uma em trajetória de sistemas didáticos do PEP, em geral, complexo em relações entre os sistemas didáticos que o constituem.

Parafraseando Chevallard (2005), a construção de um tipo de resposta R pode ser vista como uma trajetória de sistemas didáticos em diacronias sincrônicas, no sentido da diacronia dos sistemas didáticos atenderem uma sincronia de MEPR, eliminando as rotas sem saída, os fracassos, mas conservando toda a riqueza do desenvolvimento fecundo do PEP considerado.

\section{Uma trajetória como construção de uma resposta: análise e resultados}

Nesta pesquisa, é considerado como objeto de ensino do professor, que é um dos autores e que designaremos por $\mathbf{P}$, a resolução de equações do segundo grau para responder a questão $Q_{0}$ expressa do seguinte modo: $O$ que ensinar sobre a equação do segundo grau e como ensinar?

Essa questão emerge após o estudo da TAD e do problema da desarticulação das praxeologias escolares evidenciado por essa teoria, frente às práticas docentes do professor $\mathrm{P}$ sobre o ensino da resolução das equações do segundo grau em que adotava um MEPRo descrito pela seguinte sequência, que denotamos por $\mathrm{ST}_{0}$ :

$\mathrm{T}_{01}$ - Definir uma equação do $2^{\circ} \mathrm{grau}$.

Toda equação do tipo $a x^{2}+b x+c=0$, com $a, b$ e $c$ sendo números reais e $a \neq 0$, é uma equação do $2^{\circ}$ grau.

$\mathrm{T}_{02}$ - Exemplificar equações do $2^{\circ} \mathrm{grau}$;

$\mathrm{T}_{03}$ - Identificar os coeficientes $\mathrm{a}$, b, c de uma equação;

$\mathrm{T}_{04}$ - Classificar as equações do $2^{\circ}$ grau como completa ou incompleta;

As técnicas são apresentadas por meio de exemplos.

$\mathrm{T}_{05}$ - Resolver a equação incompleta do tipo $a x^{2}-c=0 ; x^{2}-25=0, x= \pm \sqrt{25}, x=$ $\pm 5$

$T_{06}$ - Resolver a equação incompleta do tipo $a x^{2}+b x=0 ; x^{2}-5 x=0$, toma o fator comum em evidência, vem $x(x-5)=0$ e escreve que $x=0$ e $x=5$.

$\mathrm{T}_{07}$ - Resolver equações completas do $2^{\circ} \mathrm{grau} a x^{2}+\mathrm{bx}+\mathrm{c}=0 \quad$ usando a fórmula de resolução $x=\frac{-b \pm \sqrt{b^{2}-4 a c}}{2 a}$; resolver $x^{2}-11 x+28=0 . a=1, b=-11$ e $c=28$, substituindo na fórmula

$x=\frac{11 \pm \sqrt{121-112}}{2}$, donde segue $x=\frac{11 \pm 3}{2}$. E daí, as raízes $x^{\prime}=7$ e $x^{\prime}=4$ 
A praxeologia dominante do docente em questão apresenta as técnicas de resolução de equações por meio de exemplos, cada uma com sua técnica distinta, sem relações claras entre elas, o que contrasta com o preconizado pelo modelo praxeológico de Chevallard, que trataria a resolução das equações em unidade, a partir de uma clara integração de praxeologias que vivem na escola do ensino fundamental.

O enfretamento dessa problemática foi realizado por um PEP, como método de pesquisa legitimado pela $T A D$, que se constitui em unidade complexa em volume de dados e relações entre os sistemas didáticos nele construídos, que torna hercúleo o trabalho de descrevê-lo em sua totalidade. Entretanto, seus dados permitem construir uma trajetória com início no sistema didático inicial, chamado estado inicial, e por meio de sitemas didáticos intermediários, chamados de estados ordinais, até atingir o sistema didático final, ou estado final, que produz uma resposta admissível.

Assim, é apresentada a seguir uma trajetória cujos estados ordinais são tomados em sincronias, mas em acordo com a diacronia do PEP. A trajetória assim construída, que constitui uma entre outras possíveis, denominamos de trajetória em diacronias sincrônicas da TDI que fundamenta o MEPR e, consequentemente, a resposta $R$ encontrada, segundo o preconizado pelo modelo praxeológico de Chevallard sobre a articulação e integração praxeológica segundo um saber, no caso, o que contempla a resolução da equação do segundo grau.

\section{Primeiro estado da diacronia da trajetória}

A primeira obra estudada, que se mostrou como um MER que responde ao problema da desarticulação do saber em questão foi a obra de Silva e Guerra (2009) inspirada na obra, também considerada no estudo, de Queysanne e Delachet (1964) quando apresentam os passos iniciais da álgebra como teoria das equações.

O estudo das obras de Queysanne e Delachet (1964) e de Silva e Guerra (2009) mostraram, segundo o professor, que a segunda é uma releitura escolar de uma obra acadêmica, pois traduziu o objeto em questão por meio de praxeologias que vivem nas escolas atuais, como se pode notar no quadro 1.

Quadro 1: Modelo Epistemológico de Referência inicial -MERo

A equação do $2^{\circ}$ grau é apresentada na forma $a x^{2}+b x+c=0$,com $a \neq 0$, que permite escrevê-la como sendo $x^{2}+p x+q=0$, que é um polinômio do segundo grau que pode ser pensado como um produto de polinômios do $1^{\circ}$ grau: $x^{2}+p x+q=(x-\alpha)(x-\beta)$

A solução da equação $(x-\alpha)(x-\beta)=0$ pode ser encontrada observando-se que o produto de dois números reais $\mathrm{A}$ e $\mathrm{B}$ é nulo $(A B=0)$ quando um dos fatores for nulo $(A=0$ ou $B=0)$. Assim, $x-\alpha=0$ ou $x-\beta=0$, obtendo-se $x=\alpha$ ou $x=\beta$

Se $x^{2}+p x+q=(x-\alpha)(x-\beta)$, então $x^{2}-(\alpha+\beta) x+\alpha \beta$ é idêntico a $x^{2}+p x+q$. Assim,

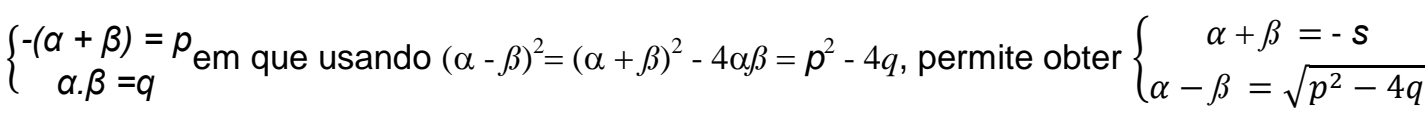
e com isso a fórmula de resolução da equação do $2^{\circ}$ grau $x=\frac{-b \pm \sqrt{b^{2}-4 a c}}{2 a}$ 
A característica de integração das praxeologias escolares acima faz o professor encaminhar um tipo de compreensão que é considerado como seu MEPR. Esse modelo epistemológico permite, então, analisar e questionar diferentes praxeologias sobre a resolução da equação do segundo grau presentes no Ensino Fundamental, e em particular, a praxelogia dominante usada pelo professor.

A partir de $M E P R_{1}$ foi construído, então, como uma tradução do MER ${ }_{0}$, uma resposta praxeológica $R_{1} q u e$ foi difundida e defendida no sistema didático auxiliar inicial $S_{1}\left(P, A, R_{1}\right)$, em que A denota alunos da quarta etapa da EJA de uma escola pública. $R_{1}$ se traduz na seguinte sequência $\left(\mathrm{ST}_{1}\right)$ :

Intenção didática: Tarefas $T_{11}$ em conexão com $T_{12}$ criam condições para encaminhar a resolução de segundo grau na $T_{13}$

$\mathrm{T}_{11}$ : Desenvolver os produtos de polinômios do tipo $(x+a)(x+b)$, para evidenciar $a$ relação entre os coeficientes do polinômio produto de segundo grau $\left(x^{2}+S x+P\right) \operatorname{com}$ os coeficientes dos fatores polinomiais do primeiro grau; $S=a+b$ e $P=a . b$.

$\mathrm{T}_{12}$ : Fatorar polinômios do $2^{\circ}$ grau com uma variável do tipo $x^{2}+S x+P$ como produto de binômios $(x+a)(x+b)$. Técnica: encontrar empiricamente $a$ e $b$ de modo que $a+b=S$ e $a \cdot b=P$.

$\mathrm{T}_{13}$ : Resolver as equações do tipo $x^{2}+p x+c=0$. Técnica por meio de exemplo, articulando e integrando as tarefas anteriores $T_{11}$ e $T_{12}$.

Intenção didática: Problematizar a técnica da tarefa com a resolução dex ${ }^{2}-2,5 x+2,5$ $=0$.

Nesse caso, as tarefas/técnicas se tornam problemáticas e mostram que $T_{11}$ e $T_{12}$ são limitadas para resolução de equações do segundo grau.

Intenção didática: Encaminhar nova técnica de resolução por meio das seguintes tarefas.

$\mathrm{T}_{14}$ : Desenvolver a diferença $(a-b)^{2}-(a+b)^{2}$, para evidenciar a relação entre quadrado da soma, quadrado da diferença entre dois termos e o produto desses termos.

$\mathrm{T}_{15}$ : Solucionar sistemas de equações do $1^{0} \mathrm{grau}$ com duas variáveis, pelo método da adição.

$\mathrm{T}_{16}$ : Resolver equações do tipo $x^{2}+S x+P=0$, para dados valores de $S$ e $P$, tomando $x^{\prime}$ $+x^{\prime \prime}=\operatorname{Se~} x^{\prime} \cdot x^{\prime \prime}=P$, por meio do sistema

$$
\left\{\begin{array}{c}
\mathrm{x}^{\prime}+\mathrm{x}^{\prime \prime}=-\mathrm{S} \\
\mathrm{x}^{\prime}-\mathrm{x}^{\prime \prime}=\sqrt{(-\mathrm{S})^{2}-4 \mathrm{P}}
\end{array}\right.
$$

$\mathrm{T}_{17}$ : Desenvolver a fórmula $x=\frac{-b \pm \sqrt{b^{2}-4 a c}}{2 a}$ da equação do $2^{\circ}$ grau $a x^{2}+b x+c=0$, por meio do sistema $\mathrm{S}_{1}$.

Essa organização, no entanto, sucumbe quando, no ensino, é confrontada pelo questionamento de um aluno quanto ao caráter empírico de adivinhação usado na técnica da tarefa $T_{13}$. Esse questionamento encaminha a problematização da técnica pelo professor que em seguida continua com sua organização por meio da tarefa $T_{14}$. No entanto, esta tarefa se mostrou estranha às suas práticas de resolução de equação do segundo grau.

Na organização matemática construída $\left(R_{1}\right)$ há alguma integração entre as tarefas $T_{15}$, $T_{16}$ e $T_{17}$, mas estas independem de $T_{14}$, ou seja, esta tarefa se mostra desnecessária. Além disso, $R_{1}$ é construída em dois blocos com intenções de evidenciar duas técnicas, a segunda com maior alcance, no entanto, não está clara a relação entre elas, talvez pelo isolamento de $T_{14}$ entre os dois blocos. 
Em resumo, não há unidade em $\mathrm{R}_{1}$ como preconiza o modelo praxeológico da TAD e presente $M E P R_{1}$. A questão $Q_{1}$, então, se põe: Como eliminar a estranheza e dar unidade a organização, respeitando de algum modo o MEPR ${ }_{1}$ ?

\section{Segundo estado da diacronia da trajetória}

A questão $Q_{1}$ deu seguimento à diacronia da TDI instaurando o sistema solitário $\quad S_{1}$ $\left(P, R_{1}\right)$ seguido de sistemas didáticos solitários e auxiliares sem respostas satisfatórias a essa questão que, considerando o espaço aqui delimitado, são omitidas.

No entanto, em um sistema didático auxiliar com o orientador (denotado por P") desse professor, representado por $\mathrm{S}_{2}\left(\mathrm{P}, \mathrm{P}\right.$ ", $\left.\mathrm{T}_{14}\right)$ o direcionou ao encontro da noção de saberes pré-existentes apresentados na obra de Chevallard (2005), mais especificamente, dos saberes protomatemáticos da escola, no sentido de serem aprendidos por imitação em situações específicas. São indispensáveis no ensino e aprendizagem das práticas matemáticas escolares, embora não sejam objetos de ensino.

O sistema didático auxiliar levou à compreensão sobre a tarefa de transformação de uma tarefa problemática em uma ou mais tarefas determinadas, ou seja, em tarefas com técnicas, que levem a uma resposta à tarefa problemática. Essa é a própria noção do PEP, que consiste em gerar uma resposta para uma questão aberta $Q_{0}$ por meio de questões determinadas $\mathrm{Q}_{1} \mathrm{Q}_{2} \ldots \mathrm{Q}_{\mathrm{k}}$.

Nesse caso, emergiu a questão $\mathrm{Q}_{2}$ : como explicitar a partir do $\mathrm{MEPR}_{1}$ a tarefa problemática que é capaz de torná-la uma tarefa determinada por meio de $\mathrm{T}_{14}$ ?

\section{Terceiro estado da trajetória}

O sistema solitário $S_{3}\left(P, Q_{2}\right)$ levou ao sistema didático solitário $S_{3}\left(P, M E P R_{1}\right)$ que encaminhou uma análise do $\mathrm{MEPR}_{1}$ e as respostas até então desenvolvidas à luz dessa compreensão que levou a resposta seguinte $R_{2}$, mas sem alterações do MEPR $R_{1}$ (Quadro 2).

A resposta $R_{2}$ preserva as tarefas: $T_{11}, T_{12}, T_{13}$ e $T_{17}$; substituindo as tarefas $T_{14}, T_{15}, T_{16}$ por $T_{13}, T_{14}$

Quadro 2 - Organização a partir de $S_{3}\left(P, M E P R_{1}\right)$.

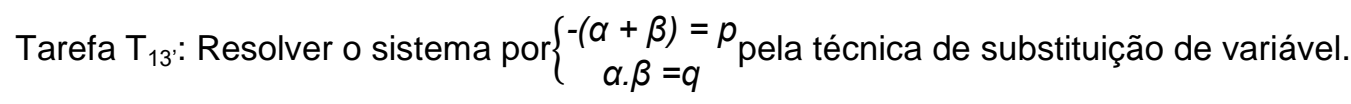

Intenção didática: Mostrar que a resolução do sistema leva para uma equação de segundo

grau do tipo $\mathrm{x}^{2}-(\alpha+\beta) \mathrm{x}+\alpha \beta=0$ e, portanto, essa tarefa de encontrar $\alpha+\beta=-p$ e $\alpha . \beta=q$, é problemática.

Tarefa $\mathrm{T}_{14}$ : Resolver o sistema anterior sabendo que $(\alpha-\beta)^{2}=(\alpha+\beta)^{2}-4 \alpha \beta=p^{2}-4 q$

Intenção didática: Resolver a tarefa problemática anterior transformando-a por meio da relação acima na tarefa determinada de resolver o seguinte sistema de duas equações pelo método

$$
\text { da adição }\left\{\begin{array}{c}
\alpha+\beta=-s \\
\alpha-\beta=\sqrt{p^{2}-4 q}
\end{array}\right.
$$

Essa resposta construída $R_{2}$ pareceu atender às intenções didáticas do professor $\mathbf{P}$ e o preconizado pela TAD quanto ao modelo praxeológico, pois há uma inequívoca integração de praxeologias onde as praxeologias antecedentes funcionam como tecnologias das praxeologias precedentes. 
Uma questão emerge no exercício de realização de $R_{2}$ em sala de aula com professores em formação ( $\left.P^{\prime}\right)$ do PARFOR (Plano Nacional de Formação de Professores), o sistema auxiliar $S_{3}\left(P, P^{\prime}, R_{2}\right)$, é a questão $Q_{3}$ : Qual o saber matemático que dá suporte ao $M E P R_{1}$ e, em consequência, a essa integração de praxeologias? Pois, esse modelo epistemológico, enquanto resposta até então encontrada, parece limitar-se ao estudo da resolução de equações do segundo grau não deixando claro o saber matemático que dá unidade às integrações das praxeologias.

\section{Quarto estado da trajetória}

Não estava clara a articulação que engendrou as integrações. Esse questionamento encaminhou o sistema didático solitário $S_{4}\left(P, Q_{3}\right)$ que levou ao sistema didático solitário $\mathrm{S}_{4}\left(\mathrm{P}, \mathrm{O}_{3}\right)$, onde $\mathrm{O}_{3}$ é obra de Lima, et al. (1998) sobre a matemática do ensino médio, que o encaminhou ao encontro do professor $\mathbf{P}$ com o Teorema Fundamental da Álgebra (TFA) e da relação dafatoração dos polinômios em fatores polinomiais do primeiro e e segundo grau e a determinação de suas raízes.

A análise da obra encaminhou a compreensão de que o saber do TFA dá unidade ao $\mathrm{MEPR}_{1}$, pois este busca encontrar as raízes a partir da fatoração do trinômio do segundo grau associado como preconizado por esse saber. Além disso, permite produzir técnicas de longo alcance, ou seja, para equações polinomiais de grau supeior a dois, além de dar uma razão matemática para o estudo das equações polinomiais do primeiro e segundo grau.

Podemos pensar o MEPR 2 como uma leitura de $\mathrm{MEPR}_{1}$ para a fatoração de um polinômio de grau n, o que insere a problemática da impossibilidade da fatorar o polinômio a partir de seus coeficientes quando n é maior que 4, mas preserva a essência de que um polinômio pode ser escrito como produto de fatores polinomiais do primeiro e segundo graus. Se isto é conhecido, a determinação das raízes é imediata, desde que saibamos encontrar as raízes das equações do primeiro grau.

Por outro lado, O MEPR 1 pode ser visto como uma interpretação do MEPR 2 para $n=2 \mathrm{e}$ nesse sentido encaminha que um trinômio $T=a x^{2}+b x+c$, pode ser escrito como $T=a\left(x^{2}+(b / a) x+c / a\right)=a(x+\alpha)(x+\beta)$ em que $\alpha+\beta=b / a$ e $\alpha \beta=c / a, \quad \alpha$ e $\beta$ reais, desde que $(\alpha-\beta)$ seja um número real. Caso contrário, o trinômio é irredutível e, nesse caso, não tem raízes reais.

No entanto, essa compreensão quando posta à prova nosistema didático auxiliar $\mathrm{S}_{4}(\mathrm{P}$, HVP, TFA), onde HVP denota a história de vida do professor sobre as práticas de fatoração e resolução de equações do segundo grau,encaminhou a seguintequestão $Q_{5}$ : Fatorar um trinômio tem a resolução da equação de segundo grau como técnica associada; ou a solução da equação do segundo grau tem a fatoração do trinômio do segundo grau como técnica associada? - que instaura o quinto estado da diacronia da trajetória.

\section{Quinto estado da trajetória}

O sistema didático solitário $S_{5}\left(P, Q_{5}\right)$ leva ao sistema didático solitário $S_{5}(P, L D E)$, onde LDE (livros didáticos escolares) denota obras didáticas usadas no ensino básico, mais precisamente, do oitavo ano e nono ano do Ensino Fundamental.

As análises dessas obras mostraram que a primeira parte da questão não faz parte do programa escolar, pois o estudo sobre a fatoração de um trinômio do segundo grau antecede o estudo de resolução das equações do segundo grau. Essa organização escolar 
do currículo de matemática implica que a técnica usada para fatoração de um trinômio do segundo grau é limitada, não permitindo responder defitivamente esse tipo de tarefa de fatoração.

Essa limitação da técnica tem levado diferentes autores de livros didáticos a considerar somente tipos particulares de trinômios, como por exemplo, em Bonjorno, et al. (2009) e Andrini e Vasconcelos (2015), que imitam a fatoração do trinômio quadrado perfeito como um tipo de tarefa inversa dos produtos notáveis como técnica; ou excluem a fatoração como objeto de ensino, como fazem, por exemplo, os livros didáticos da coleção Aprender a Viver (AGUIAR et al., 2009) que não apresenta a fatoração no seu $4^{\circ}$ volume referente à $4^{a}$ Etapa da EJA.

A segunda parte da questão, portanto, é que devia ser considerada como afirmativa, pois permite produzir técnica de longo alcance, ou seja, para equações polinômiais de grau supeior a dois, além de dar uma razão matemática para o estudo das equações polinomiais do primeiro e do segundo grau.

Esse conflito entre o $\mathrm{MEPR}_{1}$ e o preconizado pela organização do currículo escolar sugere que este modelo epistemológico seja adotado para os tipos de tarefas de fatoração do trinômio do segundo grau, considerando que recorre somente a tarefas pertinentes com o ensino de Matemática no oitavo ano, inclusive, se admitido pela escola, inserir nesteano a resolução de equações do segundo grau com a técnica de fatoração como sugere o modelo epistemológico.

O trinômio não redutível, ao contário dos redutiveis, não tem raizes reais. Essa taxionomia de redutível e não redutível, que tem implicações diretas sobre a resolução das equações do segundo grau, pode ser alcançada por meio das tarefas de produtos de polinômios, em particular sobre os produtos notáveis, e das tarefas sobre resolução dos sistemas de equações do primeiro grau estudados até o oitavo ano. O trinômio uma vez fatorado torna o encontro das raízes de modo imediato. Essa intenção didática leva a seguinte resposta $\mathrm{R}_{3}{ }_{3}$ (que denotamos aqui como a sequência $\mathrm{ST}_{5}$ ):

$T_{5(1)}$ : Usando os produtos notáveis relativos aos termos $\alpha e \beta$, transformar o sistema de equações $\left\{\begin{array}{c}\alpha+\beta=\mathrm{s} \\ \alpha^{2}-\beta^{2}=\mathrm{k}\end{array}\right.$ no sistema $\left\{\begin{array}{c}\alpha+\beta=\mathrm{s} \\ \alpha-\beta=\mathrm{u}\end{array}\right.$ e calcular $\alpha$ e $\beta$, com s e $\mathrm{k}$ conhecidos.

$T_{5(2)}$ : Usando os produtos notáveis relativos aos termos $\alpha$ e $\beta$, calcular os valores de $(\alpha$ $+\beta)^{2} e(\alpha-\beta)^{2}$ dados os valores de $\alpha^{2}+\beta^{2}$ e $\alpha . \beta$.

$T_{5(3)}$ : Usando os produtos notáveis relativos aos termos $\alpha$ e $\beta$, calcular os valores de $\alpha+\beta$ e $\alpha-\beta$, dados $\alpha^{2}+\beta^{2}$ e $\alpha . \beta$.

$\mathrm{T}_{5(4)}$ : Transformar o sistema de equações $\left\{\begin{array}{c}\alpha^{2}+\beta^{2}=\mathrm{q} \\ \alpha \cdot \beta=\mathrm{p}\end{array}\right.$ no sistema $\left\{\begin{array}{c}\alpha+\beta=\mathrm{s} \\ \alpha-\beta=\mathrm{u}\end{array}\right.$ e calcular $\alpha$ e $\beta$, sendo que p e q são dados, usando as Tarefas $T_{4(8)}$ e $T_{4(9)}$.

$T_{5(5)}$ : Usando os produtos notáveis relativos aos termos $\alpha$ e $\beta$, calcular $(\alpha-\beta)^{2}$ dados $\alpha+\beta$ e $\alpha . \beta$.

$T_{5(6)}$ : Determinar se existem ou não números reais que produzam uma dada soma e um dado produto. 
$T_{5(7)}$ : Transformar o sistema de equações $\left\{\begin{array}{c}\alpha+\beta=s \\ \alpha . \beta=p\end{array}\right.$ no sistema $\left\{\begin{array}{c}\alpha+\beta=s \\ \alpha-\beta=u\end{array}\right.$ e calcular $\alpha$ e $\beta$ (se possível), dados s e p.

$\mathrm{T}_{5(8)}$ : Fatorar, se possível, os trinômios do $2^{\circ}$ grau do tipo $T=x^{2}+S x+P$ como produto de binômios do $1^{0}$ grau do tipo $T=(x+\alpha) \cdot(x+\beta)$, em que $S=\alpha+\beta$ e $P=\alpha . \beta$.

$T_{5(9)}$ : Escrever o trinômio $T=a x^{2}+b x+c$ na forma $T=a\left(x^{2}+S x+P\right)$.

$T_{5(10)}$ : Fatorar, se possível, o trinômio $T=a x^{2}+b x+c$ como o produto $T=a(x+\alpha) \cdot(x+$ $\beta)$.

$T_{5(11)}$ : Calcular o valor da variável $x$ de modo que $(x-\alpha) \cdot(x-\beta)=0$.

$T_{5(12)}$ : Calcular o valor de $x$ de modo que $x .(x-\alpha)=0$.

$\mathrm{T}_{5(13)}$ : Calcular as raízes de trinômios do $2^{0}$ grau do tipo $T=a x^{2}+b x+c$.

A análise da resposta $R_{3}{ }_{3}$ parece eliminar a problemática da tarefa do professor de ensinar a tarefa de fatoração do trinômio de segundo grau diante de seu aluno hipotético AH. Esse aluno é construído pelo professor a partir de sua história de vida em relações com os alunos concretros na escola para engendrar um sistema didático auxiliar $S_{5}\left(P, A H, R_{3}{ }_{3}\right)$. $E$ mais, essa tarefa permite resolver as equações do segundo grau.

De qualquer modo é imprescindível que a resposta $R_{3}^{\diamond}$ seja colocada à prova em sistemas didáticos auxiliares com alunos concretos. Mas, independentes disso, outras provas podem ser encaminhadas como a da instituição docente, entendida como os outros professores de matemática da escola. Estes podem não ser convencidos da eficácia de $R_{3}$ ou mesmo vê-la com estranheza, considerando que contém tarefas que não vivem rotineiramente nos livros didáticos. Além disso, os alunos e seu entorno, como os pais e colegas de outras classes, podem não admitir o estudo dessas tarefas por, supostamente, tratarem tarefas do ano seguinte.

Entretanto, independente das restrições expostas, a resposta $\mathrm{R}_{3}{ }_{3}$ implica em questionar o currículo, no mínimo sobre a organização curricular adotada e legitimada pelos livros didáticos. Afinal, surge o seguinte questionamento $Q_{6}$ : o estudo das resoluções das equações se insere ou não nas organizações matemáticas sobre fatoração? Se afirmativo, qual a implicação sobre a técnica ensinada para a resolução de equações do primeiro grau? Ou ainda, qual a implicação para os programas de matemática do Ensino Básico, incluindo suas organizações matemáticas para o estudo, se for admitido que a resolução das equações polinomiais insere-se nas organizações sobre fatoração de polinômios?

Como se pode observar, o PEP não se encerra facilmente. Até este ponto, o MEPR do

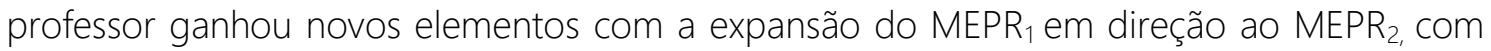
adição de novas praxeologias, e relações entre elas, como, por exemplo, as situações de transformar tarefas que surgem: tal como enfrentar tarefas cuja função não é resolver ou encontrar a solução de uma tarefa, mas transformá-la em tarefas determinadas, tarefas que já tenham sido incorporadas pelos alunos e professores para permitir encontrar sua solução.

A noção de tarefas transformadoras parece ser o cerne da questão de como construir integrações praxeológicas, pois a problematização de tarefas escolares, na instituição escolar, exige para seu enfrentamento somente as praxeologias (ou seus fragmentos) até então estudadas e incorporadas pelos alunos e professores. 
Este saber não se insere em um saber específico matemático, mas como ferramenta indispensável para as atividades matemáticas e, por isso, na escola, ganha uma função de destaque para a construção de novas organizações de praxeologias articuladas e integradas para o ensino, e, por consequência, para o exercício da profissão docente. Por que não integrá-las ao corpo de conhecimento ensinado, na escola e na formação docente?

\section{Resultados encontrados e encaminhamentos}

A construção da trajetória do PEP apresentada evidencia a mudança de relação do professor com a resolução da equação do segundo grau por meio da adição de novos elementos tecnológico-teóricos que fundamentaram seu MEPR que, em consequência, explicitou-sepor meio daconstrução de novas praxeologias.

Sob a compreensão do problema de formação anunciado por Chevallar (2009a), podemos afirmar então que o PEP engendrado pela prática de ensino do professor, entendida como compostas das duas fases da TDI, frente à reconstrução do MEPR, constitu um percurso de formação pessoal para o professor - PFPP.

A riqueza profunda do PEP pode ser influenciada de algum modo pelo objeto de ensino escolhido, que podem implicar em ganhos mais relevantes ou não de saberes e procedimentos matemáticos, acadêmicos ou escolares, para o professor, mas os encaminhamentos incertos permitem evidenciar outros saberes, às vezes, indispensáveis para as práticas matemáticas que nem sempre são objetos de ensino.

Foi evidenciado, por exemplo, o papel funcional de relações ou propriedades entre tarefas como técnicas das tarefas de transformação de uma tarefa problemática em tarefa determinada, ou seja, dotada de técnica que permite enfrentá-la. Embora as tarefas de transformações sejam rotineiras nas atividades matemáticas, elas são frequentemente recorridas no ensino, pelo professor, como bypass nas realizações das atividades matemáticas escolares. Isso é aqui observado nas relações entre expressões algébricas sobre produtos notáveis como técnicas indispensáveis para as transformações e por ele recorrida, quando a percebe, para construção de novas respostas praxeológicas.

O encontro com as tarefas de transformação na trajetória remeteu reflexivamente ao encontro de diferentes técnicas de trarefas de transformção, por exemplo, que são usadas nas práticas de ensino escolares, como as propriedades dos conjuntos numéricos em relação a uma dada operação que, geralmente, não são evidenciadas como regras para sintetizar expressões algébricas e numéricas; a substituição de variáveis em diferentes tipos de tarefas, por exemplo, como a redução dos sitemas algébricos lineares. Em todos esses casos a tarefa de transformação e suas técnicas são apresentadas por mostração, de modo naturalizado, sem questionamentos, como parte da resolução de uma tarefa problemática, e, por isso, tornadas invisíveis.

Talvez, por isso, frequentemente, o uso dos teoremas matemáticos, em articulação, ou isolados como técnica de transformação de tarefas no ensino da matemática escolar - o papel funcional de transformar uma tarefa possível de ser realizada criando as hipóteses que permitirão uma ou mais tarefas serem enfretadas, pela simples anunciação do teorema - não seja explícito, podendo ser até inexistente.

Assim, por exemplo, considerar os triângulos inscritos em um semicírculo é assegurar que são retângulos que criam a hipótese do teorema de Pitágoras, permitindo usar as 
relações métricas e trigonométricas fartamente conhecidas desse tipo de triângulo, para responder diferentes praxeologias escolares sobre esses objetos em relação.

As tarefas de tranformação e suas técnicas podem ser vistas, então, como um dispositivo para construir integrações praxeológicas, pois estas integrações podem ser descritas como a transformação de uma tarefa problemática em tarefas rotineiras, no sentido de terem sido incorporadas anteriormente. Pode-se dizer que, de algum modo, esse pensar está no centro do modelo didático proposto pela TAD: naturalizar, problematizar o alcance da técnica, e, reconstruir a técnica para um dado tipo de tarefa, para só então institucionalizar essa técnica para o tipo de tarefa considerada. Foi uma técnica de transformação de tarefas que permitiu produzir as respostas $\mathrm{R}^{\diamond}$ durante o PEP e ajudaram a traduzir o MEPR em respostas praxeológicas $R$.

Além de aquisições de novos saberes e procedimentos que resultam em novas organizações praxeológicas, O PEP engendrado pela prática de ensino, considerando as duas fases da TDI, também encaminhou de modo inevitável problemas relativos ao currículo e programas escolares para o ensino da matemática.

A complexidade e robustez dessa problemática exigem esforços que não admitem ser enfrentados por um professor isolado e se constitui em grande interesse para futuras pesquisas a serem realizadas por diferentes especialistas, mas com participação indispensável da instituição de docentes pesquisadores.

Finalmente, o PEP não seria engendrado sem saberes evidenciados pela TAD, como o problema da desarticulação das praxeologias bem como o do papel funcional tecnológico das organizações praxeológicas nas construções de novas organizações praxeológicas. Esses saberes em articulação engendrado pela TAD parece ter sido a mola propulsora para levar o professor a questionar o saber matemático, e, vê-lo como problemático para ser ensinado e, em consequência, para ser aprendido.

Esse modo de pensar, sem dúvida, provê as condições para o questionamento das práticas de ensino desde que sejam entendida como as duas fases da TDI em iteração contínua, uma de reconstrução reflexiva sobre o saber escolar a ser ensinado e outra do ensino reflexivo questionador sobre o saber reconstruído.

Portanto, as teorias do programa epistemológico, em particular, a TAD, que inclui em seu cerne a TTD e o seu dispositivo metodológico PEP, mostram-se como parte indispensável da infraestrutura de saberes úteis e indispensáveis para a formação inicial e continuada de professores de matemática.

\section{Referências}

AGUIAR, C. A. et al. Coleção Viver, Aprender. Educação de Jovens e Adultos. Segundo segmento do Ensino Fundamental. Vol. 4, $1^{\text {a }}$ edição. São Paulo, Global: Ação Educativa, 2009.

\section{ANDRADE, R. C. D. A noção de tarefa fundamental como dispositivo didático para um percurso de formação de professores: o caso da geometria. Tese (Doutorado) - Universidade Federal do Pará, Instituto de Educação Matemática e Científica, Programa de Pós-Graduação em Educação em Ciências e Matemáticas, Belém, 2012.}

ANDRINI, A, VASCONCELOS, M. J. Praticando Matemática 8. 4 ed. Renovada. Editora do Brasil, São Paulo, 2015. 
ARAYA, A. MATHERON, Y. Una problemática de la memoria: Proposiciones y ejemplos para su estudio antropológico en didáctica de las matemáticas. In: Sociedad, Escuela y Matemáticas: Aportaciones de la Teoría Antropológica do Didáctico (TAD), RUIZ, L., ESTEPA, A. GARCIA, F. J. Pág. 261-278. Universidade de Jaén, 2007.

ASSUNÇÃO, C. A. G. Práticas com Matemáticas na Educação do Campo: o caso da redução à unidade na Casa Escola da Pesca. Tese (Doutorado) - Universidade Federal do Pará, Instituto de Educação Matemática e Científica, Programa de Pós-Graduação em Educação em Ciências e Matemáticas, Belém, 2016.

BARQUERO, B. Ecología de la Modelización Matemática en la enseñanza universitaria de las Matemáticas.Tese (Doutorado) - Universitat Autónoma de Barcelona, Espanha, 2009.

BARQUERO, B., BOSCH, M. e GASCÓN, J. Ecología de la modelización

matemática:Restricciones transpositivas en las instituciones universitárias. Communication au 2e congrès TAD, Uzès 2007.

BOLEA, P. El proceso de algebrización de organizaciones matemáticas escolares. Monografía del Seminario Matemático García de Galdeano, n. 29. Zaragoza, Spain: Departamento de Matemáticas, Universidad de Zaragoza, 2003.

BONJORNO, J. R., OLIVARES, A., BONJORNO, R.A., GUSMÃO, T. Matemática: fazendo a diferença. Edição renovada. Ed. FTD, São Paulo, 2009.

BOSCH, M., CHEVALLARD, Y. GASCÓN, J. Science of Magic? The use of models and theories in didactics of mathematics. In Bosch, M. Proceedings of the 4th Conference of the European Research in Mathematics Education. 2006.

BOSCH, M.; GASCÓN, J. Fundamentación antropológica de las organizaciones didácticas: de los "talleres de prácticas matemáticas" a los "recorridos de estudio e investigación". Diffuser les mathématiques (et les autres savoirs) comme outils de connaissance et d'action, p.4985,2010. Disponível em <http://www.atd.tad.org/wapontent/upload/2012/05/marianaJosepCITAD-II-2010.pdf>

BOSCH, M.; GASCÓN, J. Las prácticas docentes del profesor de matemáticas. XlèmeÉcoled'Été de Didactique des Mathématiques, de 2001.

CHARLOT, Bernard. Da relação com o saber: elementos para uma teoria. Tradução de Bruno Magne. Porto Alegre: Artes Médicas, 2000.

CHEVALLARD, Y.; BOSCH, M.; GASCÓN, J. Estudar Matemática: o elo perdido entre o ensino e a aprendizagem. Porto Alegre: Artmed Editora, 2001.

CHEVALLARD Y. (1985) La transposition didactique - Du savoir savant au savoir enseigné, La Pensée sauvage. Grenoble, deuxième édition augmentée, 1991

CHEVALLARD, Y . Organisations didactiques: 1. les cadres généraux, 1998a. Disponível em :http://yves.chevallard.free.fr/spip/spip/IMG/pdf/Organisations_didactiques_1_1998_.pdf.A cesso em 15 Abril de 2016.

CHEVALLARD, Y. Organisations didactiques: 2. Gestes, dispositifs, programmes,1998b.

Disponível em:

<http://yves.chevallard.free.fr/spip/spip/IMG/pdf/Organisations_didactiques_2_1998_.pdf>. Acesso em 15 abril de 2016. 
CHEVALLARD, Y. Organiser l'etude. 1. Structures \& fonctions.(2002) Disponível em $<$ http://yves.chevallard.free.fr/spip/spip/article.php3?id_article=52>. Acesso em: agosto de 2015.

CHEVALLARD. Y. El análisis de las prácticas docentes en la teoría antropológica de lo didáctico. Recherches en Didactique des Mathématiques, v. 19, n. 2, p. 221-266, 1999.

CHEVALLARD. Y. La Transposición Didáctica: del saber sabio al saber enseñado. 3. ed. 2. reimp. Buenos Aires: Aique Grupo Editor, 2005.

CHEVALLARD. Y. La TAD face au professeur de mathématiques. 2009a. Disponível em: <http://yves.chevallard.free.fr/spip/spip/article.php3?id_article=162>. Acesso em: 15 maio. 2017.

CHEVALLARD. Y. La notion d'ingénierie didactique, un concept à refonder: questionnement et éléments de réponse à partir de la TAD. 2009b. Disponível em:

<http://yves.chevallard.free.fr/>. Acesso em: 24 jun. 2017.

CHEVALLARD, Y. Introduction à la théorie anthropologique du didactique / Introdução à teoria antropológica do didático. Slides bilíngue: Francês/ português.2011. Disponível em: <http://yves.chevallard.free.fr/>. Acesso em: 24 jun. 2013.

CHEVALLARD. Y.Éléments de didactique du développement durable. Leçon1. Enquête codisciplinaire \& EDD. (2012-2013). Disponível em:

<http://yves.chevallard.free.fr/spip/spip/IMG/pdf/Didactique_du_DD_2012-2013_1.pdf>. Acesso em 20 de dezembro de 2016.

CHEVALLARD, Y. Concepts fondamentaux de la didactique : perspectives apportées par une approche anthropologique. Recherches en didactique des mathématiques, 12/1, p. 73-111, 1992.

FONSECA, C. Discontinuidades Matemáticas Y Didácticas entre la Enseñanza Secundaria Y la Enseñanza Universitária. Tesis Doctoral Escuela Universitaria de Ingeniería Técnica Industrial de Vigo, Espanha, 2004.

FONSECA, C BOSCH, M.; GASCÓN, J. El momento del trabajo de la técnica en la completación de organizaciones matemáticas: el caso de la "regla de Ruffini". Revista Latinoamericana de Investigación en Matemática Educativa (RELIME), v. 22, n. 2; p.05-34, 2010.

GARCÍA, F. J.; GASCÓN, J.; HIGUERAS, L. R.; BOSCH, M., Mathematical modelling as a tool for the connection of school mathematics. ZDM,v. 38, p. 226-246, 2006.

GASCÓN, J. Fenómenos y problemas en didáctica de las matemáticas, en Ortega, T. (Editor): Actas del III Simposio de la SEIEM, Valladolid, 129-150. (1999)

GASCÓN, J. Incidencia del modelo epistemológico de las matemáticas sobre las prácticas docentes. Revista Latinoamericana de Investigación en Matemática Educativa, México, v. 4, n. 2, p. 129,159, 2001

GASCÓN, J. Efectos del autismo temático sobre el estudio de la Geometría en Secundaria I.

Desaparición escolar de la razón de ser de la Geometría. Revista SUMA, 44, 25-34. 2003. 
GASCÓN, J. Del Problem Solving a los Recorridos de Estudio e Investigación. Crónica del viaje colectivo de una comunidad científica. Revista Iberoamericana de Educación Matemática (UNIÓN), n. 22, p. 9-35, 2010.

GASCÓN, J. Las Matemáticas para la Enseñanza en una Formación del Profesorado Basada en el Estudio de Cuestiones. Bolema, Rio Claro (SP), v. 28, n. 48, p. 319-340, 2014.

GUERRA, R. B., ANDRADE, R. C. D. Tarefas Fundamentais do Fazer matemático Escolar: organização matemática para o ensino da geometria analítica. Margens (UFPA), v. 6, p. 109135, 2013.

LIMA, E.; CARVALHO, P. C. P.; WAGNER, E.; MORGADO, A. C. A Matemática do ensino médio. Rio de Janeiro: Sociedade Brasileira de Matemática, 1998. (Coleção do Professor de Matemática, v.3)

LUCAS, C., FONSECA, C., GASCÓN, J., CASAS, J. O Fenômeno Didático Institucional da Rigidez e a Atomização das Organizações Matemáticas Escolares. Bolema, Rio Claro (SP), v. 28, n. 50, p. 1327-1347, dez. 2014.

QUEYSANNE, M.; DELACHET, A. A álgebra moderna. Tradução de Gita K. Ghinzberg. São Paulo: Difusão Européia do Livro, 1964. (Coleção Saber Atual)

RAVEL, L. Des programmes a la classe: etude de la transposition didactique interne:Exemple de l'arithmétique en Terminale $S$ spécialité mathématique. Thèse préparée au sein de l'équipe de Didactique des Mathématiques (DDM), Laboratoire Leibniz-IMAG. 2003.

SIERRA, T. A. Lo matemático en la creación y análisis de Organizaciones Didácticas. El caso de los sistemas de numeración. 2006. Tese (Doutorado em out/2006) - Universidad Complutense de Madrid, 2006.

SILVA, D.P. A Invariável Prática da Regra de Três. Tese (Doutorado em Educação em Ciências e Matemáticas). Universidade Federal do Pará, Intituto de Educação Matemática e Científica. Belém, 2017.

SILVA, F. H. S.; GUERRA, R. B. Contextualização do ensino da matemática. In: SILVA, F. H. S. Formação de professores: mitos do processo. Belém: UFPA, 2009.

SILVA, R. O conhecimento matemático-didático do professor do multisseriado: análise praxeológica. Tese (Doutorado) - Universidade Federal do Pará, Instituto de Educação Matemática e Científica, Programa de Pós - Graduação em Educação em Ciências e Matemáticas, Belém, 2013. 